\title{
Monte Carlo Simulations of High-speed, Time-gated MCP- based X-ray Detectors: Saturation Effects in DC and Pulsed Modes and Detector Dynamic Range
}

\author{
Craig Kruschwitz and Ming Wu \\ National Security Technologies, LLC, Los Alamos, New Mexico 87544 \\ Ken Moy \\ National Security Technologies, LLC, Santa Barbara, California 93111
}

Greg Rochau

Sandia National Laboratories, Albuquerque, New Mexico, 87185

We present here results of continued efforts to understand the performance of microchannel plate (MCP)-based, high-speed, gated, $\mathrm{x}$-ray detectors. This work involves the continued improvement of a Monte Carlo simulation code to describe MCP performance coupled with experimental efforts to better characterize such detectors. Our goal is a quantitative description of MCP saturation behavior in both static and pulsed modes. We have developed a new model of charge buildup on the walls of the MCP channels and measured its effect on MCP gain. The results are compared to experimental data obtained with a short-pulse, high-intensity ultraviolet laser; these results clearly demonstrate MCP saturation behavior in both DC and pulsed modes. The simulations 
compare favorably to the experimental results. The dynamic range of the detectors in pulsed operation is of particular interest when fielding an MCP-based camera. By adjusting the laser flux we study the linear range of the camera. These results, too, are compared to our simulations.

\section{INTRODUCTION}

High-speed, gated x-ray detectors based on straight-channel microchannel plates are a powerful diagnostic tool for two-dimensional, time-resolved imaging and time-resolved X-ray spectroscopy. Such detectors have become standard diagnostics on fast Z-pinch experiments and laser-driven inertial confinement fusion experiments. These detectors consist of a gold photocathode coated on the rear face of the MCP, a phosphor screen coated on a fiber-optic faceplate, and a film recorder or charge-coupled device (CCD) to record the image. Detector gating is achieved by sending a subnanosecond high-voltage pulse through a microstrip transmission line coated onto the front face of the MCP. As the high-voltage pulse propagates along the strip line, MCP gating occurs wherever the voltage is applied.

In this paper we report on efforts to simulate, using Monte Carlo methods, the behavior and performance of MCP-based x-ray detectors. Specifically, we concerned ourselves with a newly designed 8-half frame MCP detector, which is similar to the detectors used in the multilayer mirror (MLM) pinhole camera diagnostic at the SNL Z machine ${ }^{1}$. Many reengineering improvements in the new NSTec camera significantly increased reliability, sensitivity, and gain uniformity. For example, by side-launching the striplines and 
changing the pusher bar design, impedance issues were solved and stress on the MCP was reduced. The etched wafer-board extends from the stripline to the feed-through, reducing the number of connections and removing the need for an epoxy fill. The improved camera had a new feature that allowed pulsed bias high voltage (HV) and monitoring its waveform on the phosphor. Decreasing potential vacuum issues and pulsebiasing the phosphor decreased the failure rate from electrically induced light contamination, as it was demonstrated in recent Z-pinch physics shots at SNL ZR facility. The MCP was purchased from Burle, Inc., which had an L/D ratio of $46(\mathrm{~L}=460 \mu \mathrm{m}$, $\mathrm{D}=10 \mu \mathrm{m}$ ), with a channel bias angle of $8^{\circ}$. Both MCP surfaces were coated using physical vapor deposition at angles of $60^{\circ}$ and $45^{\circ}$ from the surface normal, respectively. They were coated in four separate layers with the following materials and thicknesses: $75 \AA \mathrm{Cr}, 5000 \AA \mathrm{Cu}, 75 \AA \mathrm{Cr}, 1000 \AA \mathrm{Au}$. The back surface was coated uniformly. The front surface was coated in eight separated half strips, in which four long strips were $8 \mathrm{~mm}$ wide $\times 25 \mathrm{~mm}$ long, the other short strips were $8 \mathrm{~mm}$ wide $\times 22 \mathrm{~mm}$ long, separated by $1 \mathrm{~mm}$. The phosphor was coated with P43 by Lexel and ITO was coated by DRLI's 70CHTS coating procedure. The faceplate of the phosphor was Block Press BLE59-6 from Incom.

This camera is operated by sending $\sim 500$-ps flat-top pulses with different delay settings to eight half-strip-lines coated onto the MCP input face. Repeated reflections from the infinite impedance at the end of the each half-strip and the impedance mismatch at the input, broaden the voltage pulse to more than $1 \mathrm{~ns}$ full width half maximum (FWHM). 
As part of NSTec's development and characterization efforts, a large body of data was obtained for the 8-frame camera. These data include static and pulsed sensitivity data using a short pulse (sub-picosecond) high intensity UV laser (200 nm wavelength). We have also made detailed measurements of the voltage as a function of time and position along each microstrip using a picoprobe voltage probe. This large body of experimental data for the camera allows for thorough comparisons to simulations. Of interest for this paper are data dealing with the MCP dynamic range and gain saturation. Understanding this behavior is essential in order to fully comprehend the experimental data obtained using the camera.

The Monte Carlo code used in this paper has been described in a previous paper. Essentially, the code uses typical secondary electron emission probability distributions to describe the electron multiplication and cascade down a single microchannel in the MCP. The code is similar to codes used by previous authors ${ }^{2}{ }^{4}$, but contains a more detailed physical model of the cascade and amplification processes. For example, our model contains algorithms for dealing with elastic reflection of low-energy electrons from the channel wall, a requirement that the total secondary energies not exceed that of the primary electron, and a model for the suppression of secondary electron generation due to charging at the channel wall surface. Furthermore, unlike codes used by others, our simulation code is capable of dealing with time-dependent voltages. In this article we will briefly describe the Monte Carlo model and the physical models included in it. The rest of the article will then discuss the experimental data we have obtained for the NSTec/SNL 8-frame, gated, x-ray pinhole camera and compare these data to simulations. 


\section{Simulation Model}

The Monte Carlo model we used has been described previously, and is similar to the models put forth by previous researchers. The model simulates the emission of secondary electrons and their dynamics in a single channel. It is assumed that cross talk effects between channels are negligible. Also included in the model are the effects of elastically scattered electrons. This is particularly important for low-energy electrons and is essential for properly describing the MCP response across the broad range of voltage biases we are interested in. The model also had an energy conservation requirement that precludes the total secondary electron energy from exceeding that of the primary electron. The model also included space charge effects arising from the high numbers of electrons that can be present in the channel in some situations, and secondary gain suppression arising from channel wall charge buildup.

\section{Secondary Electron Emission Equations}

A simulation is begun by specifying a mean number of initial electrons, henceforth called primary electrons. These are assumed to be generated by interactions of x-rays or UV photons with the reduced lead glass channel surface. The actual number of primary electrons is sampled from a Poisson distribution.

The directions in which the primary electrons are emitted are given by a pair of angles for each electron. One of these is defined relative to the surface normal and is assumed to 
obey a cosine distribution. The other is an azimuthal angle sampled from a uniform distribution between 0 and $2 \pi$, with 0 being towards the top of the channel (the input surface of the MCP). The initial energies of the primary electrons are somewhat more difficult to determine and are expected to be different for x-ray and UV sources. For $\mathrm{x}$-rays, we assume the energies are sampled from the following probability distribution ${ }^{5}$

$$
f\left(E_{s}\right)=C \cdot \exp \left\{-\frac{\left[\ln \frac{E_{s}}{E_{0}}\right]^{2}}{2 \sigma^{2}}\right\},
$$

where $E_{0}=2.5 \mathrm{eV}$, the most probable energy, and $\sigma=0.65$. As will be discussed momentarily, this is the same probability distribution that we use to determine secondary electron energies. This is used because we lack data on the energy distributions of electrons produced from reduced lead glass by x-rays. The values for $E_{0}$ and $\sigma$ have been chosen to match experimental data ${ }^{6}$ on MCP glass secondary emission. For UV photons, the energy is determined by the work function of the reduced lead glass material of the MCP and the UV photon energy, which is $6 \mathrm{eV}$ in our experiments. The work function of the reduced lead glass is poorly known, but values of around $5 \mathrm{eV}$ have been quoted in the literature ${ }^{7}$. We assume that photoelectrons produced by the UV photons have an initial energy between $0.8-1 \mathrm{eV}$. This value was chosen primarily to match the data.

With the relevant parameters of the primary electrons fully determined, the simulations proceeds by calculating the trajectories of the electrons in the channel under the influence of the applied voltage. Each electron is determined to either collide with the channel wall or leave the channel. If an electron collides with the channel wall, the energy with which 
it impacts, $V_{i}$, is calculated and the angle at which it impacts is determined. The probability with which it is elastically reflected from the channel wall is given by ${ }^{8}$

$$
\delta_{e l}=\frac{\left(\sqrt{V_{i}+V_{0}}-\sqrt{V_{i}}\right)^{2}}{\left(\sqrt{V_{i}+V_{0}}+\sqrt{V_{i}}\right)^{2}},
$$

where $V_{0}$ is an unknown parameter chosen to best fit the data. We find that a value $\sim 160$ eV fits our data quite well.

If the electron is not reflected then the mean number of secondary electrons generated in the collision is given by ${ }^{9}$

$$
\delta\left(V_{i}, \theta_{i}\right)=\delta_{m}\left(\theta_{i}\right) \frac{s V_{i} / V_{m}\left(\theta_{i}\right)}{s-1-\left(V_{i} / V_{m}\left(\theta_{i}\right)\right)^{s}},
$$

where $s$ is a free parameter with value greater than 1 chosen to best fit the data, $\theta_{i}$ is the impact angle (relative to the surface normal), and $V_{m}\left(\theta_{i}\right)$ and $\delta_{m}\left(\theta_{i}\right)$ are given by the following equations ${ }^{10}$

$$
\begin{gathered}
V_{m}\left(\theta_{i}\right)=V_{m}(0) / \sqrt{\cos \theta_{i}}, \\
\delta_{m}\left(\theta_{i}\right)=\delta_{m}(0) \exp \left[\alpha\left(1-\cos \theta_{i}\right)\right],
\end{gathered}
$$

where $V_{m}(0)$ is the impact energy yielding the maximum mean secondary electron yield, $\delta_{m}(0)$, at normal incidence, and $\alpha$ is a constant to be determined by the data, typically between 0.4 and 0.6 .

The number of secondary electrons is determined by sampling from a Poisson distribution with a mean of $\delta_{m}\left(\theta_{i}\right)$. The secondary electrons are assigned initial energies by sampling from the probability distribution given in equation (1). The initial directions are given by a pair of angles for each secondary electron, exactly as for the primary 
electrons. One determines the direction relative to the surface normal and obeys a cosine distribution. The other determines the direction relative to the channel axis with all directions assumed equally likely. With energies and directions of newly produced secondary electrons fully determined their trajectories are determined by continuing the process. These procedures are followed until all electrons either leave the channel or the cascade dies out, producing zero gain.

\section{Pulsed vs. Static Voltage Bias}

The program we have developed is able to simulate MCP response to both static and pulsed voltage biases. Static voltage bias is simply handled, as the value of the voltage does not change during the cascade of electrons. Furthermore, we assume, following the work of Gatti ${ }^{11}$, that the electric field is parallel to the channel axis for static voltages. This is assumed not to be true for pulsed voltages, where the field is taken to be perpendicular to the faces of the $\mathrm{MCP}^{11}$.

Time dependence of the voltage pulse is easily included in the simulation: when a secondary electron is created, the value of the voltage at the time of creation (understood to be the same as the time of the collision of the parent electron with the channel wall) is determined and the electron's trajectory is calculated using that voltage. This approximation should be reasonable if the time scale over which the voltage changes is less than the typical time of flight of an electron in the channel, typically 5 to $10 \mathrm{ps}$. For the voltage pulses we investigated this assumption held. 


\section{MCP Gain Saturation}

Various mechanisms for MCP saturation are possible, including strip current limitations, space charge effects arising from high numbers of electrons in a given channel, and effects due to charge buildup on the channel walls. We included both space charge and wall charging effects in our simulation model.

As a result of the electron multiplication process there is a buildup of positive charge on the walls of the channel. The rate at which the lost electrons are replaced by the bias current is very small (the time scale is on the order of milliseconds) and so this is ignored. The result of the positive charge buildup is the creation of a positive potential barrier that secondary electrons created near the surface of the MCP must overcome. Those with insufficient energy to overcome this barrier remain trapped within the glass. The suppression of secondary electron emission from insulators due to charge buildup

resulting from electron bombardment has been studied by many authors ${ }^{12}{ }^{13}$. While these authors did not study MCP glass, the basic principle should be applicable to electron generation in an MCP.

We include this effect in our simulations by evaluating the amount of charge buildup and estimating the resulting potential barrier for each new generation of secondary electrons. Secondary electrons that have energies less than the estimated value of the surface potential are assumed not to escape the glass and, hence, make no contribution to the 
multiplication process. Our simulations indicate that this effect becomes important when electron numbers approach $10^{5}$, a result in good agreement with previous work ${ }^{14}$. Space charge effects are included by approximating the effects of the electron cloud on the trajectories of the electrons in the channel. We find that these effects are small and that the wall-charging effects appear to be the dominant factor in describing MCP gain saturation.

\section{Short-pulse UV Laser Experiments}

The characterization of MCP detectors was conducted at the Short-pulse Laser Facility at NSTec Livermore Operations, which provided 200-nm laser light with a 150-200 fs pulse width at $150-200 \mu \mathrm{J}$ energy per pulse. The laser beam can be expanded to cover the entire MCP detector. The uniformity of the laser beam was achieved by a homogenizer and diffuser and the laser flux was adjusted by a set of neutral density filters mounted on a wheel. Sensitivity and saturation effects of MCP in DC mode were measured by varying DC bias voltages and laser fluxes. MCP saturation effect in pulsed mode was studied by varying laser fluxes when the laser pulse and $\mathrm{HV}$ pulse overlaped at positions of interest. The timing jitter in the experiments was about 25 ps or less. Five images were recorded in each delay setting.

A negative DC bias voltage or HV pulse was applied to each strip, and the phosphor was held by a $2 \mu \mathrm{s},+3000 \mathrm{~V}$ pulse with respect to the MCP back surface. The coherent fibers plug $(36 \mathrm{~mm}$ high $\times 40 \mathrm{~mm}$ wide and 6 in. long) was used to couple the phosphor and the 
CCD camera. The glass fiber was $10 \mu \mathrm{m}$ in diameter, and the overall quality area is $34 \times$ $38 \mathrm{~mm}$. The CCD camera was a Spectral Instruments 800 series with a KAF-16801E class 2 chip. The KAF-16801E was a high-performance, monochrome area, CCD image sensor with $4096 \mathrm{H} \times 4096 \mathrm{~V}(9-\mu \mathrm{m})$ photoactive pixels.

In the DC mode, averaged sensitivity was an average number of eight strips of three laser shots in each DC bias setting and laser flux. Each image was scaled according to the laser energy of each shot. While in the pulsed mode, averaged sensitivity was an average intensity over an area of interest in a selected strip of five laser shots in each time delay setting.

\section{Comparison of Data with Simulations}

We now compare results from the short-pulse UV laser experiments described in the previous section with our efforts to simulate MCP response in the appropriate experimental situations. We begin by comparing simulated MCP sensitivities with static voltage bias data and then proceed to results using time-dependent voltages.

For the static bias voltage experiments, each of the eight strips on the MCP was held at the same voltage. The voltage bias ran from 400 to 900 VDC in 50-volt increments. The laser power was then adjusted using a series of filters, so that the experiment was performed over a two order of magnitude range of fluxes from $\sim 27 \mathrm{~nJ} / \mathrm{cm}^{2}$ to $\sim 1400$ $\mathrm{nJ} / \mathrm{cm}^{2}$, corresponding to about $10^{4}-10^{6}$ photons/channel. In this way we were able to study the MCP's response to static voltages in saturated and unsaturated regimes. 
In the simulations, the effect of increasing the laser power was approximated by increasing the mean number of electrons initiating the cascade. A true comparison would require knowledge of the quantum efficiency of the MCP for 200-nm photons. We do not have this data, but given the very large number of photons/channel per laser pulse we conclude that it must be very low $(<0.1 \%)$ for the MCP to see no indication of saturation at the lower fluxes. Lacking such knowledge, comparisons of simulations to data are done on the basis of relative increase in initial electron numbers. This assumes that the mean number of primary electrons scales linearly with the laser flux. Thus we perform simulations with the mean number of primary electrons varying between 1 and 1000 .

The experimental data are shown in Figure 1. The experimental data clearly show appreciable saturation at higher voltages for photon fluxes greater than $7 \mathrm{e} 4$ photons/channel, but saturation at lower fluxes is absent. There is, however, a fairly steep drop-off in the sensitivity for voltages below $\sim 550 \mathrm{~V}$; this drop-off is not present for higher photon fluxes. It is also clear that the general trend of sensitivity (gain) with voltage changes somewhat as the laser power changes. As the laser flux is increased the gain becomes slightly less dependent on voltage, changing from about a $G \sim V^{11}$ dependence to more nearly a $G \sim V^{9}$ dependence before the onset of saturation. The simulation results comparing raw electron numbers versus voltage are shown in Figure 2. Similar to the experimental data, the simulations exhibit clear gain saturation at high voltages when the mean number of primary electrons is between 100 and 1000. It is clear that there is a rather sharp cutoff at $\sim 10^{5}$ electrons at which the gain curve exhibits a clear plateau. Similar behavior can be seen in the raw experimental data in Figure 1. 
Experimental data and simulations results have been plotted together in Figure 3, with all data scaled to have the same value at $550 \mathrm{~V}$. The general trend of sensitivity with voltage for simulation and experiment agree reasonably well. Unfortunately, we do not know the number of electrons being extracted from the $\mathrm{MCP}$, so a quantitative comparison is not possible, but the qualitative agreement is promising. The simulations even exhibit a similar trend of decreased dependence of gain with voltage, though far less pronounced. This could imply that this effect may arise from high electron numbers present in the channel at a given time, even if the MCP is not necessarily operating at a particularly high gain.

It is of great importance for the fielding of the 8-frame camera on the ZR machine to know the effective dynamic range of the camera in pulsed mode. Figure 4 shows the voltage pulse versus time applied to a typical strip of the MCP. Additionally, a +200 VDC static bias was applied, putting the peak voltage applied around $700 \mathrm{~V}$. To test the dynamic range of the camera, the timing delay between the laser pulse and the voltage pulse was fixed to the delay, giving the maximum signal from the strip in question. Once this delay was fixed, the laser flux was increased. Ideally, the laser flux is increased until the camera response is no longer linear. Unfortunately, the maximum laser flux applied was still in the camera's linear regime, so we were unable to fully test the dynamic range. However, the data provide useful information on the camera and allow some interesting comparisons with the simulation code. Figure 5 shows the dynamic range data obtained. The camera response remains linear throughout the range of laser powers shown. This is perhaps somewhat surprising given that under static voltage bias conditions the MCP 
saturated at much smaller laser power at similar voltage. This is most likely due to two factors: 1) the difference between static and pulsed behavior - the peak voltage in the pulse is only achieved for a relatively short time; and 2) the amount of uncertainty in the timing in the experimental system.

We attempted to approximate the dynamic range experiments using the simulation code by starting a cascade at the time on the voltage pulse that produced the highest eventual yield of output electrons (this time is slightly before the voltage peak occurs). The number of output electrons was then tallied. To simulate the effect of increasing the laser power, the number of primary electrons was increased. This was done until the relationship of output electrons to primary electrons ceases to be linear. The results of the simulations are shown in Figure 6. The response is linear up to approximately 500 primary electrons. This result appears to be consistent with the DC simulation results. Thus, with this particular pulse and DC offset, the simulations imply the camera should have a dynamic range from $10^{2}$ to $10^{3}$. The experimental data indicate it may in fact be somewhat greater, but the aforementioned difficulties with interpreting the data make it uncertain.

\section{Summary}

We have discussed recent work regarding characterizing the performance and behavior of a MCP-based, time-gated, x-ray camera. We have described results of experiments performed using an intense, short-pulse UV laser, and attempts to simulate the MCP 
behavior using a Monte Carlo simulation code we developed. The experiments were performed with a static and pulsed voltage bias on the MCP, and in saturated and unsaturated regimes. The Monte Carlo code is capable of modeling both static and timedependent voltages, and contains models for describing MCP gain saturation. Thus, comparisons between the experimental data and the simulations provide a useful check on the simulations and may help broaden our understanding of MCP behavior, which is essential to understanding the data obtained by such cameras.

Comparisons between the experimental data and the simulations show that the Monte Carlo code matches the sensitivity vs. voltage trend reasonably well. The wall-charging algorithm in the code also replicates the qualitative aspects of MCP saturation. While a direct comparison of the laser power to electron number is not possible at this time, the results of both the simulations and the experimental data indicate that the camera has a dynamic range of $10^{2}$ to $10^{3}$ in pulsed mode.

${ }^{1}$ B. Jones, C. Deeney, A. Pirela, C. Meyer, D. Petmecky, P. Gard, R Clark, and J. Davis, Rev. Sci. Instrum. 75, 4029 (2004).

${ }^{2}$ A. J. Guest, Acta Electronica 14, 79 (1971).

${ }^{3}$ M. Ito and K. Oba, IEEE Trans. Nucl. Sci. NS-31, 408 (1984).

${ }^{4}$ Y. S. Choi and J. M. Kim, IEEE Trans. Elec. Dev. 47, 1293 (2000).

${ }^{5}$ J. J. Scholtz, D. Dijkkamp, and W. A. Schmitz, Philips. J. Res. 50, 375 (1996).

${ }^{6}$ A. Authinarayanan and R. W. Dudding, Adv. Electron. Electron Phys. A 40, 167 (1976). 
${ }^{7}$ A. E. Melamid, J. Appl. Spect. 16, 351 (1972).

${ }^{8}$ R. Cimino, I. R. Collins, M. A. Furman, M. Pivi, F. Ruggiero, G. Rumolo, and

F. Zimmerman, Phys. Rev. Lett. 93, 014801 (2004).

${ }^{9}$ M. A. Furman and M.T.F. Pivi, Phys. Rev. ST AB 5, 124404-1 (2002).

${ }^{10}$ H. Bruining, Physics and Applications of Secondary Electron Emission (Pergamon

Press, London, 1954)

${ }^{11}$ E Gatti, K. Oba, and P. Rehak, IEEE Trans. Nucl. Sci. NS-30, 461 (1983).

${ }^{12}$ J. Cazaux, J. Appl. Phys. 85, 1137 (1999).

${ }^{13}$ J. Cazaux, Eur. Phys. J. AP 15, 167 (2001).

${ }^{14}$ Otto L. Landen, Perry M. Bell, John A. Oertel, J. J. Satariano, and David K. Bradley, Ultrahigh- and High-Speed Photography, Videography, and Photonics '93, Bellingham, WA, 2-13, (1993), edited by Paul W. Roehrenbeck SPIE.

This manuscript has been authored by National Security Technologies, LLC, under Contract No. DE-AC52-06NA25946 with the U.S. Department of Energy. The United States Government retains and the publisher, by accepting the article for publication, acknowledges that the United States Government retains a non-exclusive, paid-up, irrevocable, world-wide license to publish or reproduce the published form of this manuscript, or allow others to do so, for United States Government purposes. 


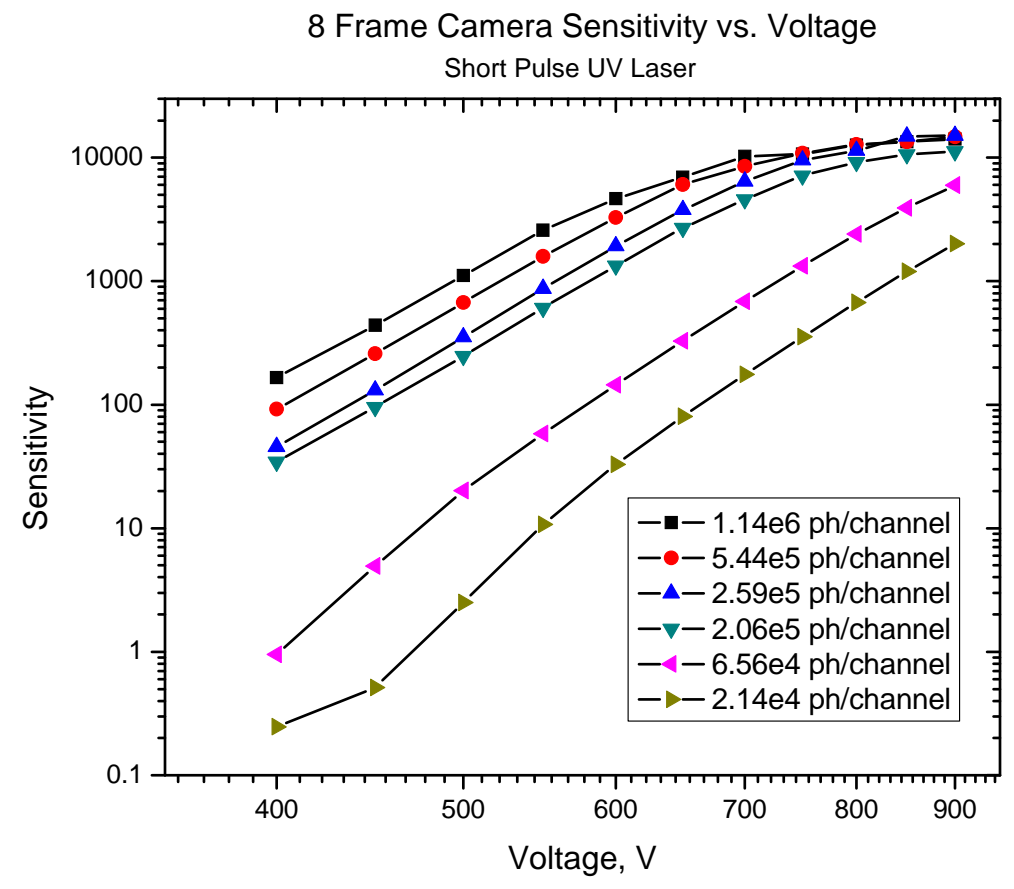

Figure 1. Sensitivity vs. voltage results for static voltage bias from intense, shortpulse UV laser (200-nm wavelength). The approximate laser flux in photons per channel is indicated. 


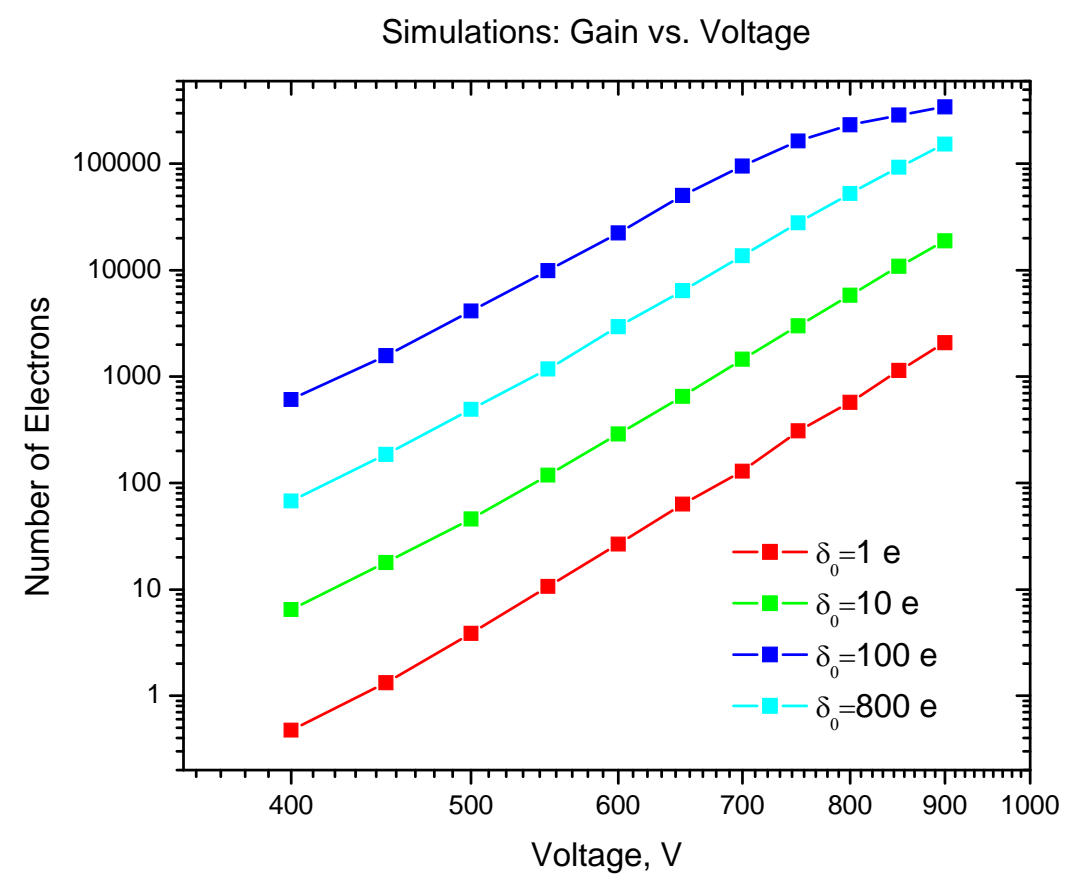

Figure 2. Simulations of electrons out vs. voltage for static voltage bias. Number of mean primary electrons is indicated. The simulations exhibit clear gain saturation for electron numbers greater than $10 \wedge 5$. 


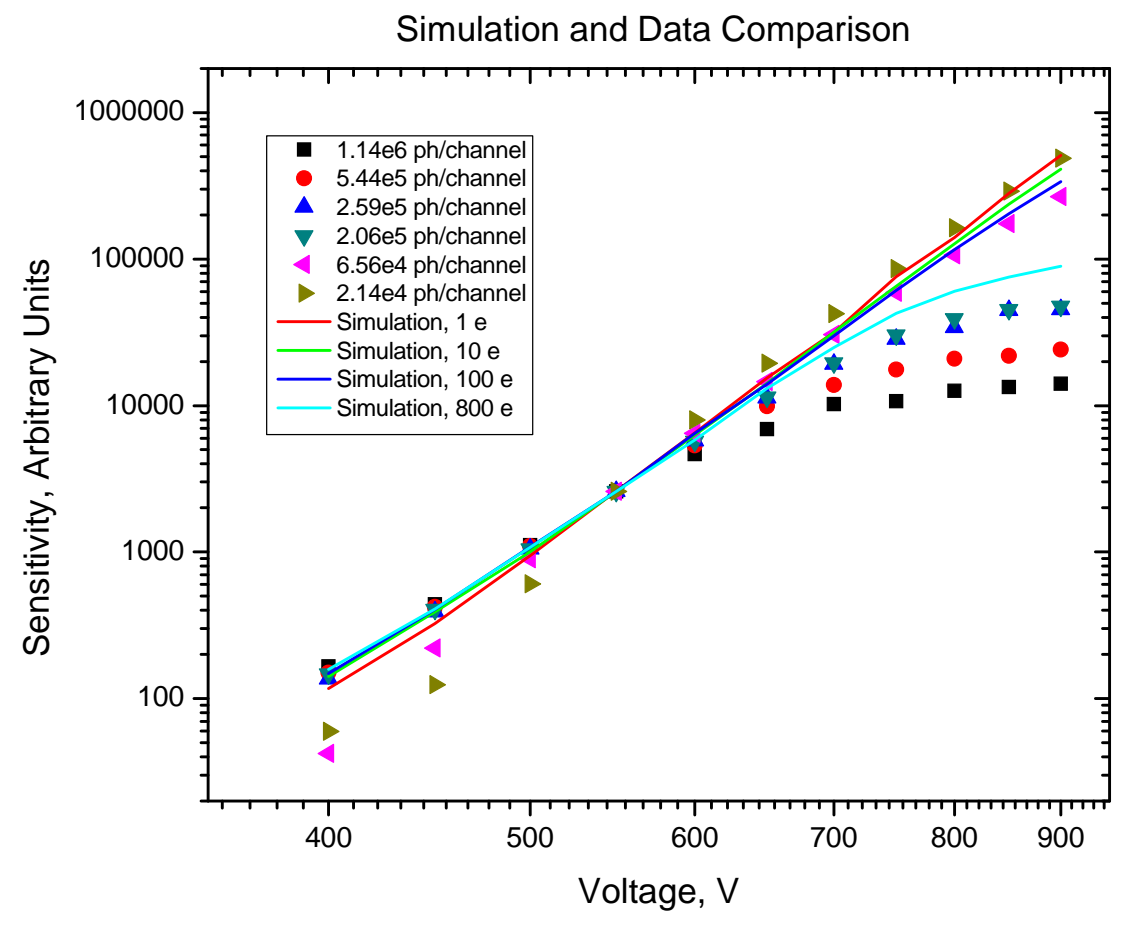

Figure 3. Comparison of simulations and experimental data. All data and simulations scaled to be equal for $550 \mathrm{~V}$. 


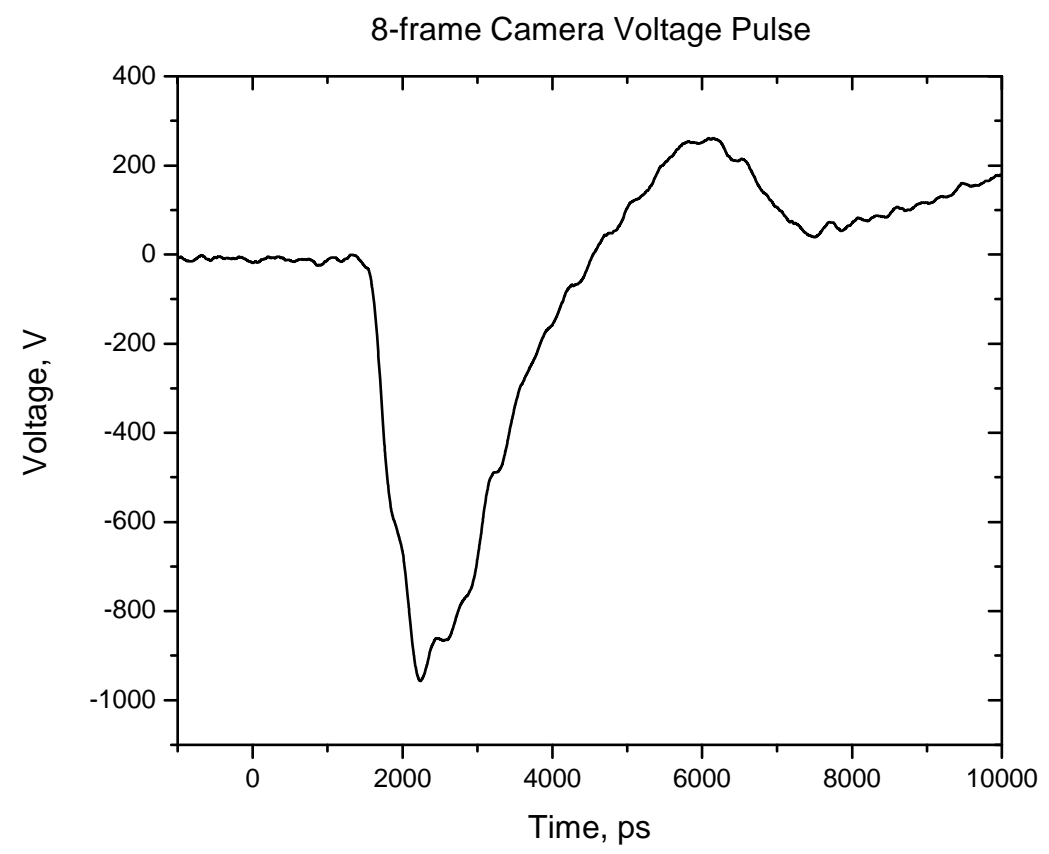

Figure 4. Voltage pulse used for time-gating the 8-frame camera. In the experiments performed here, a +200 VDC bias was used. The pulse is from a Kentech high-speed pulser. 


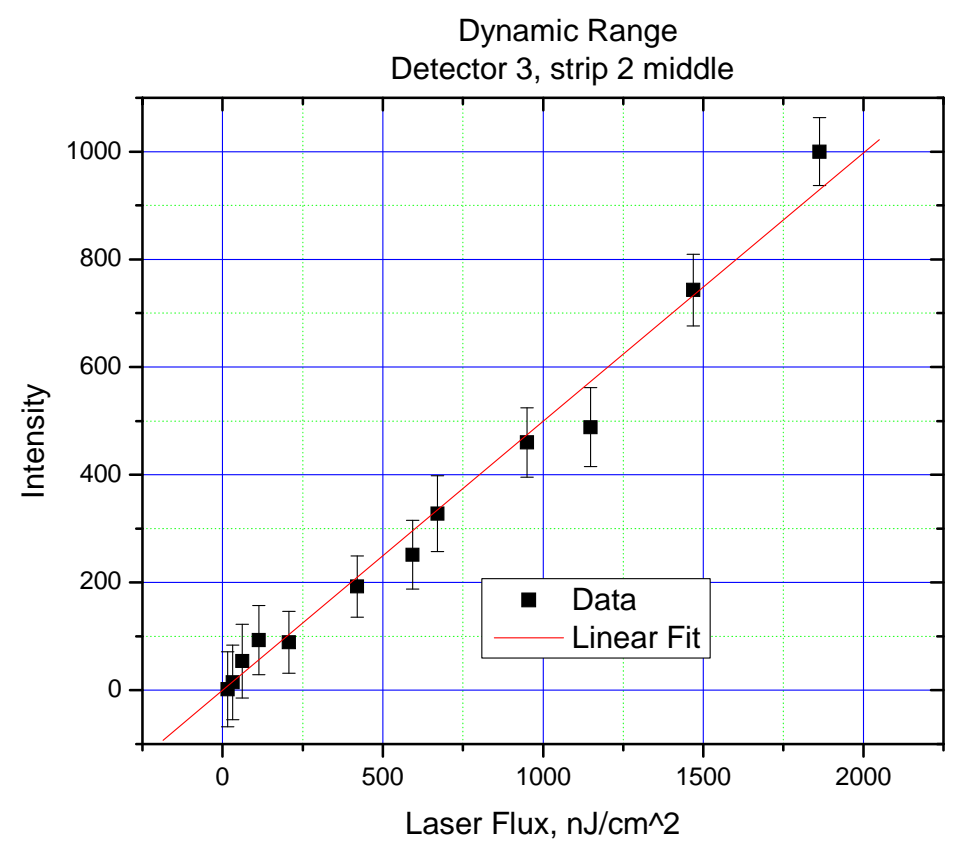

Figure 5. Data from pulsed dynamic range experiments. The detector response remains linear for the entire range of laser powers investigated. 


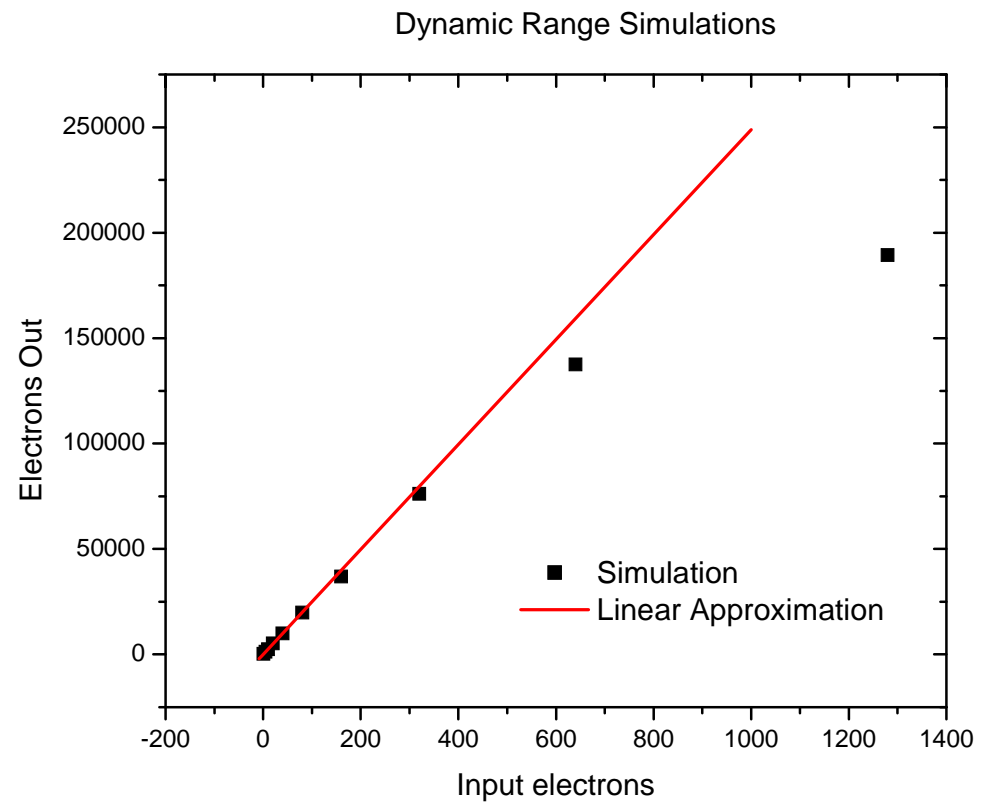

Figure 6. Results of simulations of pulsed detector dynamic range. The simulations indicate a dynamic range of $10 \wedge 2$ to $10^{\wedge} 3$. 